\title{
OPERATORS ON BANACH ALGEBRA VALUED FUNCTION SPACES
}

\author{
JOR-TING CHAN
}

\begin{abstract}
A bstract. Let $S$ be a locally compact Hausdorff space and let $A$ be a Banach algebra. Denote by $C_{0}(S, A)$ the Banach algebra of all $A$-valued continuous functions vanishing at infinity on $S$. Properties of bounded linear operators on $C_{0}(S, A)$, like multiplicativity, are characterized by Choy in terms of their representing measures. We study these theorems and give sharper results in certain cases.
\end{abstract}

\section{Introduction}

Let $S$ be a locally compact Hausdorff space and let $A$ be a Banach algebra. The Banach space (under the supremum norm) of all $A$-valued continuous functions vanishing at infinity on $S$ will be denoted $C_{0}(S, A)$. When $A=\mathbb{C}$, we simply write it as $C_{0}(S)$. The dual and bidual of $C_{0}(S, A)$ are denoted by $C_{0}^{\prime}(S, A)$ and $C_{0}^{\prime \prime}(S, A)$ respectively. Let $B(S)$ be the $\sigma$-algebra of all Borel subsets of $S$. To every bounded linear operator $T: C_{0}(S, A) \rightarrow C_{0}(S, A)$ there corresponds a finitely additive operator valued mearsure $m: B(S) \rightarrow L\left(A, C_{0}^{\prime \prime}(S, A)\right)$ such that $T f=\int_{s} f d m$ for all $f \in C_{0}(S, A)$. (See for example [1]). This is called the representing measure of $T$. If $f$ is a complex function on $S$ and if $x \in A, f \otimes x$ denote the function given by $f \otimes x(s)=f(s) x$ for every $s \in S$. When $e \subseteq S$, $1_{e}$ is the characteristic function of $e$. Let $e \in B(S)$ and $x \in A, 1_{e} \otimes x$ can be

Received September 2, 1991; revised March 20, 1992.

1980 Mathematics Subject Classification (1985 Revision). Primary 47B38; Secondary $46 \mathrm{G} 10$.

Keywords and phrases. Representing measures, multiplicative operators,multiplicatively symmetric operators, Arens product. 
viewed as an element in $C_{0}^{\prime \prime}(S, A)$ by $\left(1_{e} \otimes x\right) \mu=\int_{e} x d \mu$. Here $\mu \in C_{0}^{\prime}(S, A)$ is . identified as an $A^{\prime}$-valued measure on $S$. We have $T^{\prime \prime}\left(1_{e} \otimes x\right)=m(e) x$. One may ask how properties of $T$ are reflected in the representing measure $m$. This has been the subject matter of papers like [3] and [4] by Choy. Now $C_{0}(S, A)$ is a Banach algebra under the pointwise multiplication. The biduals $A^{\prime \prime}$ and $C_{0}^{\prime \prime}(S, A)$ are again Banach algebras under the Arens product as defined in Bonsall and Duncan [2, pp. 106-107]. The Arens product will play an important role in the subsequent discussion. However we shall refer readers to [2] and use the notations and properties given in the book without making any further reference.

The main result of [3] states that a bounded operator $T$ is multiplicative, i.e. $T$ satisfies $T(f g)=(T f)(T g)$ for all $f, g \in C_{0}(S, A)$, if and only if the representing measure $m$ satisfies $m\left(e_{1} \cap e_{2}\right)(x y)=\left(m\left(e_{1}\right) x\right)\left(m\left(e_{2}\right) y\right)$ for all $x, y \in$ $A$ and $e_{1}, e_{2} \in B(S)$. Writing $e_{1}=\left(e_{1} \backslash e_{2}\right) \cup\left(e_{1} \cap e_{2}\right)$ and $e_{2}=\left(e_{2} \backslash e_{1}\right) \cup\left(e_{1} \cap e_{2}\right)$, it can be easily verified that this latter condition is the same as

(1) $\left(m\left(e_{1}\right) x\right)\left(m\left(e_{2}\right) y\right)=0$ whenever $e_{1} \cap e_{2}=\phi$, and

(2) $m(e)$ is multiplicative for all $e \in B(S)$.

In the next section we prove that a bounded operator $T$ satisfies condition (1) above if and only if $(T f)(T g)=0$ whenever $f g=0$. We also prove that when $A$ is unital, condition (2) alone ensures that $T$ is multiplicative.

In the last section we turn to multiplicatively symmetric operators (defined below) and answer in negative a question raised in [4].

\section{Multiplicative operators}

Theorem 2.1. The operator $T$ has the property that $f g=0$ implies $(T f)(T g)$ $=0$ if and only if its representing measure $m$ satisfies $\left(m\left(e_{1}\right) x\right)\left(m\left(e_{2}\right) y\right)=0$ whenever $e_{1} \cap e_{2}=\phi$.

Proof. (Necessity) Let $x, y \in A$ and let $e_{1}, e_{2} \in B(S)$ such that $e_{1} \cap e_{2}=\phi$. For every $\mu \in C_{0}^{\prime}(S, A)$,

$$
\left(m\left(e_{1}\right) x\right)\left(m\left(e_{2}\right) y\right)(\mu)=T^{\prime \prime}\left(1_{e_{1}} \otimes x\right) T^{\prime \prime}\left(1_{e_{2}} \otimes y\right)(\mu)=\int_{e_{1}} x d T^{\prime}\left[T^{\prime \prime} 1_{e_{2}} \otimes y, \mu\right] .
$$


Let $\epsilon>0$. Choose a compact subset $e_{1}^{\prime} \subseteq e_{1}$, an open set $e_{1}^{\prime \prime} \supseteq e_{1}^{\prime}$ and an $f \in C_{0}(S)$ satisfying $0 \leq f \leq 1,\left.f\right|_{e_{1}^{\prime}}=1$ and $f=0$ outside $e_{1}^{\prime \prime}$ such that

$$
\left|\int_{e_{1}} x d T^{\prime}\left[T^{\prime \prime} 1_{e_{2}} \otimes y, \mu\right]-\int_{S} f \otimes x d T^{\prime}\left[T^{\prime \prime} 1_{e_{2}} \otimes y, \mu\right]\right|<\epsilon .
$$

Now $\int_{S} f \otimes x d T^{\prime}\left[T^{\prime \prime} 1_{e_{2}} \otimes y, \mu\right]=\left[T^{\prime \prime} 1_{e_{2}} \otimes y, \mu\right](T f \otimes x)=\int_{e_{2}} y d T^{\prime}<\mu, T f \otimes x>$. Take a compact subset $e_{2}^{\prime} \subseteq e_{2}$, an open set $e_{2}^{\prime \prime} \supseteq e_{2}^{\prime}$ and a $g \in C_{0}(S)$ such that

$$
\left|\int_{e_{2}} y d T^{\prime}<\mu, T f \otimes x>-\int_{S} g \otimes y d T^{\prime}<\mu, T f \otimes x>\right|<\epsilon .
$$

We have

$\int_{S} g \otimes y d T^{\prime}<\mu, T f \otimes x>=<\mu, T f \otimes x>(T g \otimes y)=\mu((T f \otimes x)(T g \otimes y))$. Now $f$ and $g$ can be chosen to satisfy $(f \otimes x)(g \otimes y)=0$ and hence $\mu((T f \otimes$ $x)(T g \otimes y))=0$. Since $\epsilon$ is arbitrary, we have $\left(m\left(e_{1}\right) x\right)\left(m\left(e_{2}\right) y\right)=0$. (Sufficiency) Suppose that $f, g \in C_{0}(S, A)$ satisfy $f g=0$. For every $\epsilon>$ 0, by considering the sets on which $f$, respectively $g$, are nonzero, and their relative complements from each other, we can find disjoint subsets $e_{1}, \cdots, e_{l}$, $e_{l+1}, \cdots, e_{m}, e_{m+1}, \cdots, e_{n} \in B(S)$ and $x_{1}, \cdots, x_{m}, y_{l+1}, \cdots, y_{n} \in A$ such that

$$
\left\|f-\sum_{i=1}^{m} 1_{e_{i}} \otimes x_{i}\right\|<\epsilon,\left\|g-\sum_{j=l+1}^{n} 1_{e_{j}} \otimes y_{j}\right\|<\epsilon \text { and }
$$

$\left(1_{e_{i}} \otimes x_{i}\right)\left(1_{e_{j}} \otimes y_{j}\right)=0$ for every $i=1, \cdots, m$ and $j=l+1, \cdots, n$.

Then $\left(m\left(e_{i}\right) x_{i}\right)\left(m\left(e_{j}\right) y_{j}\right)=0$ and therefore

$$
\begin{aligned}
& \|T f T g\| \\
& \left.=\|\left(\sum_{i=1}^{m} m\left(e_{i}\right) x_{i}+\left(T f-\sum_{i=1}^{m} m\left(e_{i}\right) x_{i}\right)\right)\right)\left(\sum_{j=l+1}^{n} m\left(e_{j}\right) y_{j}+\left(T g-\sum_{j=l+1}^{m} m\left(e_{j}\right) y_{j}\right)\right) \| \\
& <\epsilon\|T\|^{2}(\|f\|+\|g\|+\epsilon) .
\end{aligned}
$$

Since $\epsilon$ arbitrary, $T f T g=0$. 
Regarding the condition (2) described in the introduction, we first show that this property alone is not enough to guarantee that $T$ is multiplicative.

Example 2.2. Let $S=\left\{s_{1}, s_{2}\right\}$ be a two point set and identify $C(S, A)$ with $A \times A$. We shall construct multiplicative operators $\varphi, \psi: A \rightarrow A$ such that $\varphi+\psi$ is multiplicative while $(\varphi x)(\varphi y) \neq 0$ for some $x, y \in A$. Then $T: C(S, A) \rightarrow C(S, A)$ defined by $T(x, y)=(\varphi(x)+\psi(y), 0)$ has representing measure $m$ given by $m\left(\left\{s_{1}\right\}\right)(x)=(\varphi(x), 0)$ and $m\left(\left\{s_{2}\right\}\right)(x)=(\psi(x), 0)$. So $m$ satisfies condition (2), but by Theorem $2.1, T$ is not multiplicative. Let $A$ be $\mathbb{C}^{3}$ with a multiplication defined by $(a, b, c) \cdot\left(a^{\prime}, b^{\prime}, c^{\prime}\right)=\left(0,0, a b^{\prime}-b a^{\prime}\right)$. Under the usual $l^{2}$-norm, $A$ is a Banach algebra. Let $\varphi$ be the identity map on $A$ and $\psi$ be given by $\psi(a, b, c)=(0, a, 0)$. Then $\varphi+\psi$ is multiplicative and $\varphi(1,0,0) \psi(1,0,0)=(0,0,1)$.

When $A$ is unital we have

Theorem 2.3. If $A$ is a unital Banach algebra, then $T: C_{0}(S, A) \rightarrow$ $C_{0}(S, A)$ is multiplicative if and only if its representing measure $m$ has the property that $m(e)$ is multiplicative for every $e \in B(s)$.

Proof. We need only prove the sufficiency. Suppose that $e_{1}$ and $e_{2}$ are disjoint subsets in $B(S)$, we claim that $\left(m\left(e_{1}\right) x\right)\left(m\left(e_{2}\right) y\right)=0$ for every $x, y \in A$. Let $i$ be the unit element in $A$. Then $m\left(e_{1}\right) i, m\left(e_{2}\right) i$ and $m\left(e_{1}\right) i+m\left(e_{2}\right) i$ are all idempotents. It follows that $\left(m\left(e_{1}\right) i\right)\left(m\left(e_{2}\right) i\right)+\left(m\left(e_{2}\right) i\right)\left(m\left(e_{1}\right) i\right)=$ 0 . Multiplying both sides from the left by $\left(m\left(e_{1}\right) i\right)$ yields $\left(m\left(e_{1}\right) i\right)\left(m\left(e_{2}\right) i\right)+$ $\left(m\left(e_{1}\right) i\right)\left(m\left(e_{2}\right) i\right)\left(m\left(e_{1}\right) i\right)=0$. If we multiply the same element on the right, we get $\left(m\left(e_{1}\right) i\right)\left(m\left(e_{2}\right) i\right)\left(m\left(e_{1}\right) i\right)+\left(m\left(e_{2}\right) i\right)\left(m\left(e_{i}\right) i\right)=0$. Hence $\left(m\left(e_{1}\right) i\right)\left(m\left(e_{2}\right) i\right)-$ $\left(m\left(e_{2}\right) i\right)\left(m\left(e_{1}\right) i\right)=0$. Thus we have $\left(m\left(e_{1}\right) i\right)\left(m\left(e_{2}\right) i\right)=0$. So for every $x, y \in A$,

$$
\left(m\left(e_{1}\right) x\right)\left(m\left(e_{2}\right) y\right)=\left(m\left(e_{1}\right) x\right)\left(m\left(e_{1}\right) i\right)\left(m\left(e_{2}\right) i\right)\left(m\left(e_{2}\right) y\right)=0 .
$$

Theorem 2.4. If $A$ is a unital $C^{*}$-algebra or if $A$ is a commutative $C^{*}$ algebra, then $T$ on $C_{0}(S, A)$ is a *-algebra homomorphism if and only if $m(e)$ is 
a *-algebra homomorphism for every e $\in B(S)$.

Proof. By [3, Theorem 3.3], $T$ is involution preserving if and only if $m(e)$ is involution preserving for every $e \in B(S)$. Together with Theorem 2.3, the result follows in the unital case. In case $A$ is a commutative $C^{*}$-algebra, it suffices to prove that if $m(e)$ is a *-algebra homomorphism for every $e \in B(S)$, $\left(m\left(e_{1}\right) x\right)\left(m\left(e_{2}\right) y\right)=0$ whenever $e_{1}$ and $e_{2}$ are disjoint sets in $B(S)$ and $x, y \geq 0$. Using the fact that $m\left(e_{1}\right), m\left(e_{2}\right)$ and $m\left(e_{1}\right)+m\left(e_{2}\right)$ are multiplicative, we get $\left(m\left(e_{1}\right) x\right)\left(m\left(e_{2}\right) y\right)+\left(m\left(e_{2}\right) x\right)\left(m\left(e_{1}\right) y\right)=0$. But from our assumption on $m$, the elements $m\left(e_{1}\right) x, m\left(e_{2}\right) y$ are positive. So are $\left(m\left(e_{1}\right) x\right)\left(m\left(e_{2}\right) y\right)$ and $\left(m\left(e_{2}\right) x\right)\left(m\left(e_{1}\right) y\right)$. It follows that $\left(m\left(e_{1}\right) x\right)\left(m\left(e_{2}\right) y\right)=0$.

\section{Multiplicatively symmetric operators}

A bounded linear operator $T: C_{0}(S, A) \rightarrow C_{0}(S, A)$ is said to be multiplicatively symmetric if $T(f T g)=T((T f) g)$ for every $f, g \in C_{0}(S, A)$. In [4, Theorem 2.2] Choy proved that a one-to-one linear operator $T$ is multiplicatively symmetric if and only if $\left(1_{e_{1}} \otimes x\right)\left(m\left(e_{2}\right) y\right)=\left(m\left(e_{1}\right) x\right)\left(l_{e_{2}} \otimes y\right)$ for every $e_{1}, e_{2} \in B(\operatorname{supp} m)$ and $x, y \in A$. He asks whether the conclusion still holds if $T$ is not assumed to be one-to-one. We show that the assumption is essential.

Example 3.1. Let $A$ be $\mathbb{C}^{2}$ with the corrdinatewise multiplication and let $S$ be any compact Hausdorff space. A function $f \in C(S, A)$ can be identified in the obvious way as an ordered pair $\left(f_{1}, f_{2}\right)$ with $f_{1}, f_{2} \in C(S)$. Define $T$ by $T\left(f_{1}, f_{2}\right)=\left(0, f_{1}\right)$. Then $T$ is multiplicatively symmetric but not one-to-one. We have $\operatorname{supp}(m)=S$. But if we let $e_{1}=e_{2}=S, x=(1,2)$ and $y=(2,1)$, Then $\left(1_{e_{1}} \otimes x\right)\left(m\left(e_{2}\right) y\right)=(0,4) \neq(0,1)=\left(m\left(e_{1}\right) x\right)\left(1_{e_{2}} \otimes y\right)$.

It may be desirable to have a description of multiplicatively symmetric operators in terms of their representing measures, but we have not been able to obtain a reasonably simple formula. 


\section{References}

[1] I. Batt and E. J. Berg, "Linear bounded transformations or the spates of continum functions," J. Funct. Anal. 4(1969), 215-239.

[2] F. F. Bonsall and J. Duncan, "Numerical Ranges of Operators on Nonned Spaces and of Elements of Normed Algebras," London Math. Soc. Lecture Note Series, vol. 2, Cambritge Univ. Press, London-New York, 1971.

[3] S. T. I. Choy, "Integral representation of multiplicative, involution preserving operators in $\mathcal{L}\left(C_{0}(S, A), B\right)$," Proc. Amer. Math. Soc. 83(1981), 54-58.

[4] -, "One-to-one operators on function spaces," ibid 87(1983),691-694.

Department of Mathematics, University of Hong Kong, Hong Kong. 